\title{
Road grade estimation for look-ahead vehicle control using multiple measurement runs
}

\author{
Per Sahlholm ${ }^{\mathrm{a}, \mathrm{b}, *}$, Karl Henrik Johansson ${ }^{\mathrm{b}}$ \\ a Scania CV AB, SE-151 87 Södertälje, Sweden \\ ${ }^{\mathrm{b}}$ ACCESS Linnaeus Centre, Royal Institute of Technology (KTH), SE-100 44 Stockholm, Sweden
}

\section{A R T I C L E I N F O}

\section{Article history:}

Received 16 March 2009

Accepted 16 September 2009

Available online 30 October 2009

\section{Keywords:}

Road traffic

Global positioning systems

Kalman filters

Sensor fusion

Automotive system identification and

modeling

General automobile/road-environment

strategies

\begin{abstract}
A B S T R A C T
Look-ahead cruise controllers and other advanced driver assistance systems for heavy duty vehicles require high precision digital topographic road maps. This paper presents a road grade estimation algorithm for creation of such maps based on Kalman filter fusion of vehicle sensor data and GPS positioning information. The algorithm uses data from multiple passes over the same road to improve previously stored road grade estimates. Measurement data from three test vehicles and six experiments have been used to evaluate the quality of the obtained road grade estimate compared to a known reference. The obtained final grade estimate compares favorably to one acquired from a specialized road grade measurement vehicle with a DGPS receiver and inertial measurement unit, with an average root mean square error of $0.17 \%$ grade.
\end{abstract}

(c) 2009 Elsevier Ltd. All rights reserved.

\section{Introduction}

The economic development of the world is driving a continuing increase in the demand for goods transportation. Environmental concerns together with competitive pressure to increase efficiency make any technology that shows a potential for reductions in energy consumption highly interesting. The increase in road transportation also intensifies demand for new safety systems, to protect road users in increasingly complex traffic environments. One area that shows promise to both improve road safety and reduce energy consumption in vehicles is electronic advanced driver assistance systems (ADAS). Sensors, which are a part of many such systems, help the driver by improving the total perception of the environment. In the case of look-ahead systems, a map with stored information is used to extend the perception horizon beyond what either the driver or conventional on-board sensors can see. Actuators connected to ADAS improve vehicle control by acting in situations where the driver is unable to do so. In map-based look-ahead systems the automatic action can be based on information that the driver will only be able to get at a later time.

An increasing number of vehicle control systems utilize stored information from a map, to aid the driver in piloting the truck in a safe and economical manner. Examples of map attributes that are

\footnotetext{
* Corresponding author. Fax: +46855381395.

E-mail addresses: per.sahlholm@scania.com, per.sahlholm@gmail.com, per.sahlholm@ee.kth.se (P. Sahlholm), kallej@ee.kth.se (K. Henrik Johansson).
}

commonly used are speed restrictions, road class, road curvature and road grade. Knowledge of the current and future road grade can be used in engine and gearbox control systems to help meet the instantaneous power demand while keeping fuel consumption and environmental impact as low as possible.

The heavy duty vehicle (HDV) in Fig. 1 will speed up when going down one hill, and loose speed when climbing the next one. If the road grade for the kilometer or so directly ahead of the vehicle is known, it is possible to automatically adjust the speed in advance of up- and downhill road segments and thus save fuel without increasing trip time. The preview road grade information can also be utilized when determining if a gearshift should be performed or the state of some energy buffer changed. Furthermore the brake management system could use the road grade information to determine the highest allowable speed when going down a hill. Thus waste heat generation in excess of the system's ability to release it can be avoided. This in turn ensures that the vehicle retains emergency stopping power, a clear safety benefit.

Road grade maps of sufficient accuracy to support automatic vehicle control systems are currently not widely available. Commercial efforts to create such maps are underway, but access to them will most certainly be associated with some cost. HDVs commonly travel the same routes frequently, and are thus ideally suited to be their own probes and estimate the road grade for the small subset of all roads that are relevant for a particular vehicle.

Information about the current state of the vehicle is acquired through various on-board sensors. Information about factors that will influence the vehicle in the future cannot generally be sensed 
directly. However, a map with stored sensor readings or estimated quantities can provide the required look-ahead information and enable new control algorithms to improve overall vehicle performance. A sensor reading or estimate recorded at the current position in one run along the road can be used as look-ahead information in the next run. In order to use the map the vehicle needs to be able to position itself, this is most commonly solved by installing a satellite navigation system receiver. Fortunately, those are both cheap and commonplace nowadays.

One road characteristic which lends itself to estimation using standard HDV on-board sensors and recording in a map is the road grade. If a road is driven frequently, many estimates of the road grade can be obtained, and these can be used to increase confidence in the created map. This paper proposes such a method for road grade estimation and investigates its performance when applied to real measurements. The method has been evaluated experimentally using the three types of HDVs shown in Fig. 2.

\subsection{Problem formulation}

The problem studied in this paper is how to estimate the road grade of roads that are frequently traveled by a HDV, based on sensors that are part of the standard vehicle equipment. The vehicle is described by a vector field. $f_{v}$ defines the longitudinal movement and links the road grade to the engine torque. The road is modeled with one state for the altitude, whose dynamics are described by $f_{z}$, and one for the road grade. The total system model is

$\frac{d v}{d s}=f_{v}\left(v, \alpha, T_{\mathrm{e}}\right)$

$\frac{d z}{d s}=f_{z}(\alpha)$

$\frac{d \alpha}{d s}=0$

where $s$ is the distance along the road, $v$ is the vehicle speed, $\alpha$ is the road grade, $T_{\mathrm{e}}$ is the engine torque, and $z$ is the absolute altitude of the vehicle.

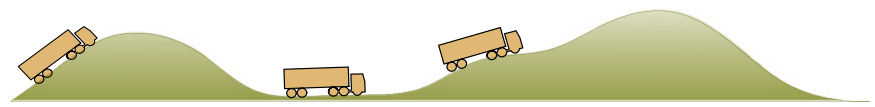

Fig. 1. HDVs traveling on a hilly road. At the position of the leftmost vehicle, it is advantageous to lower the speed to take full advantage of the upcoming downhill road segment. In the second position the overall fuel economy can be improved by increasing the speed before the steep part of the hill is reached. In the third position it is important to maintain the driving torque, to avoid costly loss of turbo pressure when entering the continued ascent.
The system model and sensor information are used together to create the road grade estimator shown in Fig. 3. The estimator uses a Kalman filter with the time-varying system model to obtain a first grade estimate. Rauch-Tung-Striebel smoothing is then applied to increase the measurement information used and avoid filter lag. The smoothed estimate is then used to update a stored map based on the relative reliability of the latest estimate and the already stored data.

The problem that is solved in this paper is how to use sensor signals already available in many HDVs to iteratively create road grade maps based on all the sensor information available from many runs along the same roads.

\subsection{Notation}

The term road grade will be used in this work to refer to the rate of change in the road surface altitude along the direction of travel for the road. In the mathematical models the road grade is expressed as an angle between the roadway and the horizontal plane, measured in radians. It is common, e.g. on road signs, to express road grade in terms of percent. This generally refers to an altitude difference divided by a corresponding traveled distance. It

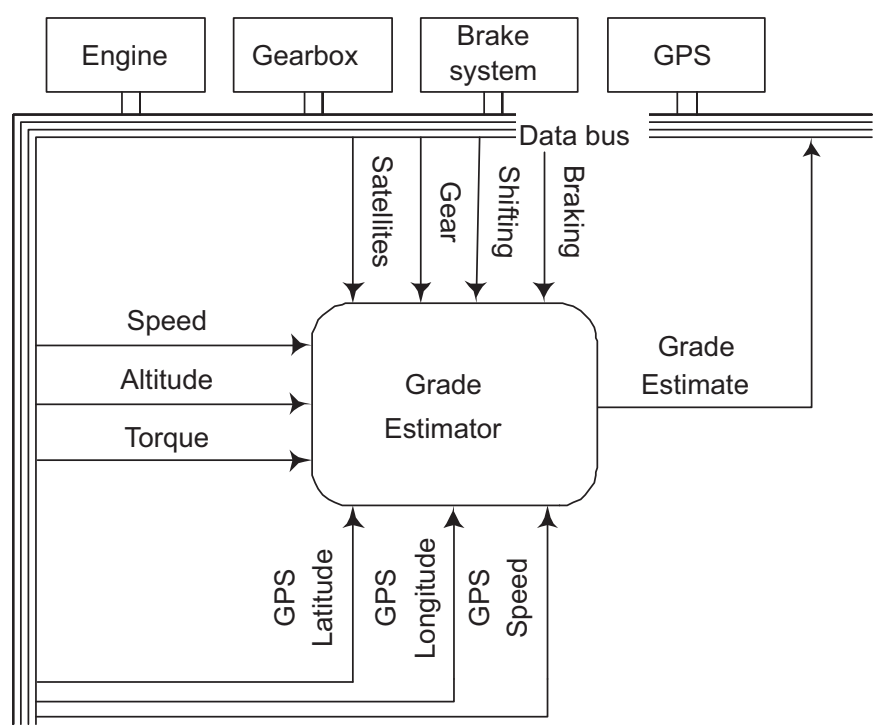

Fig. 3. The studied problem is how to estimate the road grade based on sensor information from a standard HDV. The presented estimator depends on two directly measured states, the vehicle speed and road altitude. The engine torque is treated as a measured input signal. The estimator also relies on auxiliary information about when braking and gearshifts occur, the currently engaged gear and the number of tracked GPS satellites. Finally, additional GPS signals are recorded to enable fusion of road grade estimates from multiple runs along a road. a

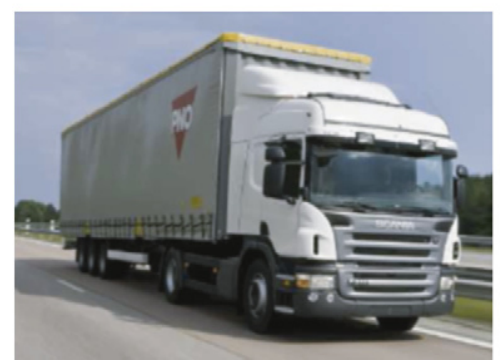

b

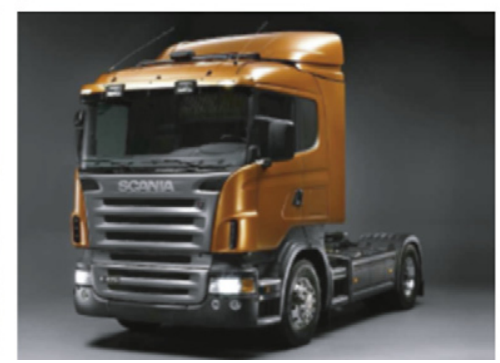

C

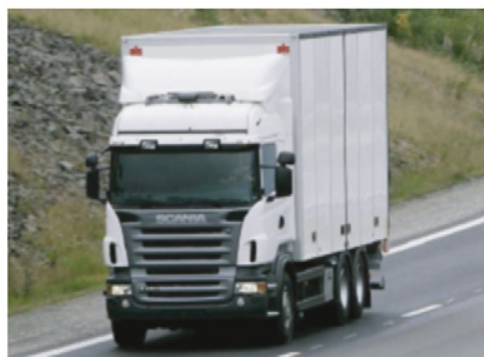

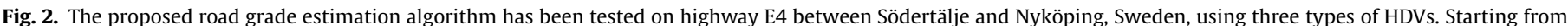
the left they were a tractor-semitrailer combination (A), tractor only (B), and rigid truck (C). (Photographs courtesy of Scania CV AB.) 
is sometimes ambiguous whether the traveled distance is measured along the incline, or if the distance along a virtual horizontal reference plane should be used. The difference in practice is very small, it does not reach $1 \%$ of the road grade until a grade of approximately $14 \%$. In this work all road grade results are presented as percent, calculated as the ratio of altitude change divided by the covered distance in the horizontal plane. Expressing changes or intervals of a quantity expressed with the unit \% is somewhat delicate. One have to make the distinction between a $5 \%$ change from a grade of $2.0 \%$ to $2.1 \%$ and a 5 percentage points change from $2.0 \%$ to $7.0 \%$. To make this clear the term "\% grade" will be used as shorthand whenever the intended meaning is a change denoted as percentage points of road grade.

\subsection{Outline}

The rest of this paper is organized as follows. Section 2 includes a motivation for the project and references to related works. The system model used in the road grade estimator is developed in Section 3. Section 4 describes the sensors used and the filtering and data fusion steps taken to arrive at the final road grade map. An account for the experimental results achieved is given in Section 5. The paper is concluded in Section 6.

\section{Related work}

Methods for estimating the road grade as well as uses for the information have been studied for some time. This section outlines various estimation schemes and important applications that have been previously described.

\subsection{Road grade estimation}

A multitude of methods for estimating the road grade can be found in the literature. In many cases the instantaneous road grade at the vehicle position is the primary objective in the estimation but various methods intended for mapping of entire road segments have also been described. As a general rule methods for estimation of the instantaneous road grade in production vehicles includes system cost as an important parameter. Contributions focused on mapping applications generally utilize expensive hardware that enables accurate results in a single pass over the road. The approach described herein, where many measurement from low-cost sensors are merged into a road grade map is quite rare.

One common approach for estimation of the instantaneous road grade is to use a sensor to directly measure the grade. A direct road grade sensor for automotive use is described in a patent application filed as early as 1971 by Gaeke (1973). More recent contributions often employ GPS receivers of various kinds to obtain road grade estimates. Bae, Ruy, and Gerdes (2001) compares a grade estimation method based on a GPS receiver with 3D velocity output and one using a two-antenna GPS. Another effort based on high precision GPS equipment is described in Han and Rizos (1999), where a road in Australia has been surveyed using geodesy GPS receivers with stationary base stations for improved accuracy. A spatial Kalman filter with height and road grade states is used to post-process the data. All these methods rely heavily on the existence of a high-quality GPS signal. Additionally, neither of the approaches is believed to work particularly well with the low cost GPS receivers that can be anticipated to be standard equipment in road vehicle in the coming few years.
The idea of using vehicle sensor information in combination with a longitudinal road model to find the road grade has been explored in Lingman and schmidtbauer (2001), where a Kalman filter is used to process a measured or estimated propulsion force or estimated retardation force and a measured velocity into a road grade estimate. A similar method, where the grade is estimated using a "recursive least squares" method based on a simple motion model has been suggested by Vahidi, Stefanopolou, and Peng (2005).

On-line road grade estimation, without GPS, based on accelerometers, calculated driveline torque and a vehicle model, or other on board sensors is state-of-the-art in today's vehicles. One proposed method and a survey of the area can be found in Fathy, Kang, and Stein (2008).

The idea of automatic creation of road maps from GPS traces has been described in a few places. An interesting approach based on position logs from many runs along a road, using consumer grade GPS receivers, is described in Schroedl, Wagstaff, Rogers, Langley, and Wilson (2004). Another automatic road map generation approach, based on more expensive DGPS receivers, is detailed in Brüntrup, Edelkamp, Jabbar, and Scholz (2005). The emphasis in this project is on the data mining methods being applied. Both these contributions treat 2D-maps without road grade information. They also do not explore the possibility to use a vehicle model and on-board sensors to improve accuracy.

An attempt to automatically identify route conditions accurately enough to be used in look-ahead vehicle control applications such as predictive powertrain control for hybrid electric vehicles is described in Carlsson, Baumann, and Reuss (2008).

The iterative road grade estimation scheme described in this paper was first introduced in Sahlholm, Jansson, Kozica, and Johansson (2007). Initial data from the highway experiments were presented in Sahlholm, Jansson, and Johansson (2007). Further developments to the method, and a more detailed study of the benefits of merging data from multiple experiments were given in Sahlholm, Jansson, and Johansson (2008). The most comprehensive description of the efforts is given in the technical report (Sahlholm, 2008). A key innovation explored in the work described herein is the method of combining the vehicle sensors and a GPS to create a road grade map based on many runs along a particular road segment.

\subsection{Applications for road grade information}

When a highway contains segments with an uphill grade so steep that a vehicle cannot maintain the desired speed, or a downhill grade so steep that overspeeding will occur unless the brake system is used, there is a potential for decreasing the fuel consumption compared to a standard cruise controller, with unchanged trip time. In driving trials such a controller has been shown to reduce the fuel used by $3.5 \%$ (Hellström, 2007; Hellström, Ivarsson, Åslund, \& Nielsen, 2009). Similar systems are also described by Fröberg (2008) and Terwen, Back, and Krebs (2004).

In hybrid electric vehicles one of the most challenging control objectives is to keep the state of charge of the energy buffer from hitting the boundaries of its operating range. In Johannesson (2006) and Johannesson and Egardt (2007) various predictive control strategies based on stochastic models of the road grade and velocity profiles for a hybrid electric passenger car in city traffic are evaluated. A current and instructive survey of results obtained by route optimized control of hybrid electric passenger cars and HDVs can be found in Gonder (2008), while a wider survey of the current state of energy buffer management research for hybrid electric vehicles is available in Salmasi (2007). 
A HDV contains a number of auxiliary units such as an electric generator, a power steering pump, a water pump, a cooling fan, an air compressor, an oil pump, and sometimes an air conditioning unit. A common trait of these is that they operate with an energy buffer, that can potentially be controlled based on future road characteristics. The potential for energy savings from using more controllable auxiliaries is explored by Pettersson and Johansson (2006).

Automatic gearboxes stand to benefit considerably from advance knowledge of the upcoming road grade. Performing a gear shift in a HDV takes time and costs energy. If the grade profile is known, the number of gear shifts required can often be reduced.

\section{Modeling}

The first step in creating the road grade estimator is to obtain a model that links the observable signals vehicle speed, $v$, altitude, $z$, and engine torque, $T_{\mathrm{e}}$ to the road grade, $\alpha$. This section starts with a description of how a vehicle model is derived from a representation of the longitudinal dynamics of a HDV as a function of time. In order to implement the estimator a model for how the road grade signal evolves is also adopted. Next, the two sub-models are put together into a complete description of the road-vehicle system in continuous time. To facilitate merging of measurement data from multiple runs along the road the system model is then transformed into the spatial domain and discretized. The described system model is non-linear in the vehicle speed. In order to use it in a standard Kalman filter it has to be linearized, this is described in Section 3.3. In Section 3.4 the measurement equation associated with the system model is detailed. A summary of the system model concludes the section.

\subsection{System model}

A basic vehicle model that describes 1D longitudinal movement and links engine torque, vehicle speed and road grade is sufficient to support the road grade estimation. Only the relatively low frequency dynamics of the vehicle itself are of interest. Higher frequency phenomena such as driveline oscillations and torsional vibration in the propeller and drive shafts can thus be ignored. The model is developed based on straightforward mechanical relations and Newton's laws of motion. This section provides an overview of the vehicle model, more details on the various subsystems and their representation can be found in Kiencke and Nielsen (2003).

A longitudinal vehicle model is used to relate the sensor signals to the road grade. The road grade can be calculated from the model when the vehicle mass, engine torque, active gear and vehicle speed are all known. In this work the vehicle mass has been assumed known, which is reasonable in a lab setting but not in the real world. In a real system the mass will have to be estimated, which introduces an additional error source in the grade estimate. The engine torque estimate comes from the onboard engine management system and is based on fuel injector opening times. The current gear is continuously reported from the gearbox management system, and the vehicle speed is measured by standard mounted wheel speed sensors. The most important forces affecting the vehicle are shown in Fig. 4. The forces are generally time-varying, time has been left out of the equations for clarity:

$F_{\text {powertrain }}=\frac{i_{\mathrm{t}} i_{\mathrm{f}} \eta_{\mathrm{t}} \eta_{\mathrm{f}}}{r_{\mathrm{w}}} T_{\mathrm{e}}$

is the net engine force. Knowledge of the current gear yields the gear ratio $i_{\mathrm{t}}$ and the efficiency $\eta_{\mathrm{t}}$ from tables. The final gear ratio $i_{\mathrm{f}}$,

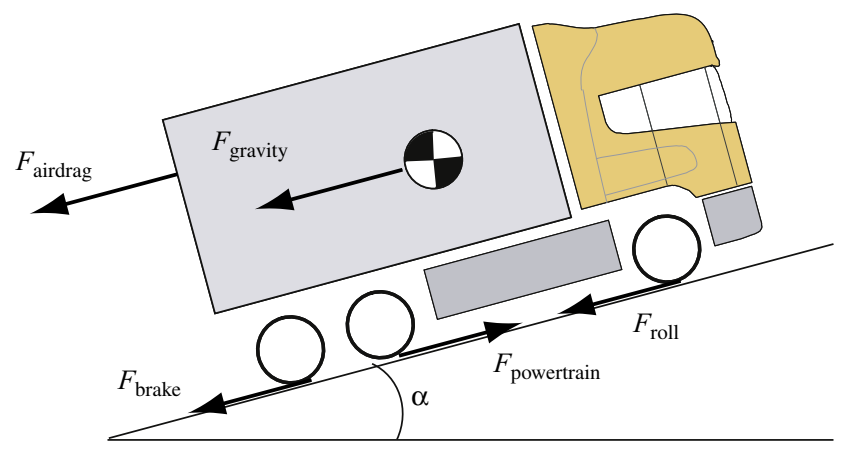

Fig. 4. Longitudinal forces acting on the vehicle.

efficiency $\eta_{\mathrm{f}}$ and wheel radius $r_{\mathrm{w}}$ are known vehicle constants. $T_{\mathrm{e}}$ denotes the engine torque:

$F_{\text {airdrag }}=\frac{1}{2} c_{\mathrm{w}} A_{\mathrm{a}} \rho_{\mathrm{a}} v^{2}$

is known through the measured vehicle speed $v$ and the constants air drag coefficient $c_{\mathrm{w}}$, vehicle frontal area $A_{\mathrm{a}}$, and air density $\rho_{\text {air }}$. A very simple model

$F_{\text {roll }}=m g c_{\mathrm{r}} \cos \alpha \approx m g c_{\mathrm{r}}$

gives the rolling resistance from the vehicle mass $m$, gravity $g$, and coefficient of rolling resistance $c_{\mathrm{r}}$. For the angles that are present on major highways the effect of $\cos \alpha$ is considerable smaller than other errors in the relatively simple vehicle model being used here. The effect of this term is therefore neglected. The road grade $\alpha$ enters the model in a much more significant way through the gravity induced force

$F_{\text {gravity }}=m g \sin \alpha$

Computing the brake force being applied to the vehicle is quite hard. A single truck or tractor unit can be attached to many different trailers at different times. These can be equipped with different types of brakes (most commonly some type of disc or drum brakes). The states of the brakes on the vehicle and its trailer are also generally unknown. The current temperature, amount of wear and weather conditions affect the efficiency of the brakes. The brake force $F_{\text {brake }}$ is thus generally unknown in a standard HDV, and is therefore excluded from the model. The influence of $F_{\text {brake }}$ is instead considered at a later stage of the method. The total dynamic vehicle mass is expressed as

$m_{\mathrm{t}}=m+\frac{J_{\mathrm{w}}}{r_{\mathrm{w}}^{2}}+\frac{i_{\mathrm{t}}^{2} i_{\mathrm{f}}^{2} \eta_{\mathrm{t}} \eta_{\mathrm{f}} J_{\mathrm{e}}}{r_{\mathrm{w}}^{2}}$

where $J_{\mathrm{w}}$ and $J_{\mathrm{e}}$ represent the inertia of the engine and the wheels, respectively. Newton's laws of motion are used to attain a differential equation describing velocity changes based on forces

$\dot{v}(t)=\frac{1}{m_{\mathrm{t}}}\left(F_{\text {powertrain }}-F_{\text {airdrag }}-F_{\text {roll }}-F_{\text {gravity }}\right)$

Changes in the road grade are assumed to be random on the time scale of the filter, which leads to the road grade model

$\dot{\alpha}(t)=0$

A GPS receiver provides a 3D position (latitude, longitude, and altitude) together with a signal indicating the number of satellites used for the position fix. The vehicle speed and the road grade are used to calculate the time derivative of the altitude and thus provide a link between the GPS and the vehicle model. The road altitude is described by

$\dot{z}(t)=v(t) \sin \alpha(t)$

Based on the described models for the vehicle on the road, and the road itself a model for the complete system can put together. A state vector $\boldsymbol{x}(t)=\left[\begin{array}{lll}v(t) & z(t) & \alpha(t)\end{array}\right]^{T}$ containing the vehicle velocity, 
the road altitude and the road slope is defined. Using (6) and the expressions presented earlier for the magnitudes of the forces involved the system model becomes

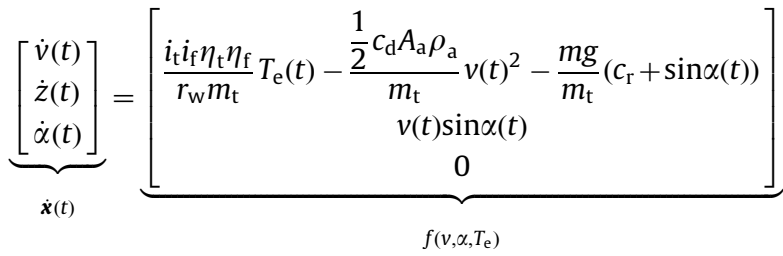

where the time dependence of the states and the input net engine torque signal has been made explicit. Then engine torque is measured and regarded as a known input signal in the model, $u(t)=T_{\mathrm{e}}(t)$. Since the transmission ratio and associated efficiency are dependent on the engaged gear the model changes discretely at gear changes. Additionally, since the driveline friction always causes an energy loss in the direction energy is flowing, the efficiencies $\eta_{\mathrm{t}}$ and $\eta_{\mathrm{f}}$ depend on whether the net engine torque $T_{\mathrm{e}}$ is positive or negative. Whenever the power flow from the engine to the wheels is negative, the efficiencies become the inverse of their nominal values, causing another discrete switch in the system model.

\subsection{Spatially sampled system model}

In order to easily obtain estimates at specific spatial locations rather than time instants a spatially sampled version of the model is derived. This is a prerequisite in order to effortlessly merge road grade estimates from multiple runs along a road.

After the change of independent variable the model is discretized using the step length $\Delta s$. By resampling measurement data from different runs along the road to represent common spatial coordinates a consistent merge can be performed. In this study the spatial sample rate was chosen to be $\Delta s=2.5 \mathrm{~m}$, since reference data with this spacing were available at the start of the project. The purpose of the model is to serve in a Kalman filter to estimate the states, so process noise to account for disturbances is added as well. One white noise process is added to each of the model states. The discretized model becomes

$$
\underbrace{\left[\begin{array}{c}
v_{k} \\
z_{k} \\
\alpha_{k}
\end{array}\right]}_{\boldsymbol{x}_{k}}=\underbrace{\left[\begin{array}{c}
v_{k-1}+\Delta s \frac{d v_{k-1}}{d s} \\
z_{k-1}+\Delta s \sin \alpha_{k-1} \\
\alpha_{k-1}
\end{array}\right]}_{f_{k}\left(\boldsymbol{x}_{k-1}, u_{k}\right)}+\underbrace{\left[\begin{array}{c}
w_{k}^{v} \\
w_{k}^{z} \\
w_{k}^{\alpha}
\end{array}\right]}_{\boldsymbol{w}_{k}}
$$

The rate of change in velocity is given by

$$
\frac{d v_{k-1}}{d s}=\frac{i_{\mathrm{t}} i_{\mathrm{f}} \eta_{\mathrm{t}} \eta_{\mathrm{f}}}{r_{\mathrm{w}} m_{\mathrm{t}}} \frac{T_{\mathrm{e} k-1}}{v_{k-1}}-\frac{\frac{1}{2} c_{\mathrm{d}} A_{\mathrm{a}} \rho_{\mathrm{a}}}{m_{\mathrm{t}}} v_{k-1}-\frac{m g}{m_{\mathrm{t}}} \frac{1}{v_{k-1}}\left(c_{\mathrm{r}}+\sin \alpha_{k-1}\right)
$$

Again, the model parameters depend on the selected gear and the direction of power flow in the driveline, making this a timevarying discrete model with traveled distance along the road as the independent variable.

\subsection{Linearized system model}

To evaluate the influence of the nonlinearity in the vehicle model a piecewise constant linear version of the model is also derived. The linear model is changed at gear changes and when the direction of power flow in the driveline changes. Each gear and power flow direction will lead to a different mode, denoted by an index $m$ added to the relevant variables. For each mode a specific torque is required to maintain a constant speed, and equilibrium in the model. The linearization point thus changes as well when the model parameters change. The linear discretized model around the equilibrium $\boldsymbol{x}_{m}$ is given by the system transition matrix $\boldsymbol{F}_{m}$ and the input matrix $\boldsymbol{G}$ according to

$\tilde{\boldsymbol{x}}_{k}=\boldsymbol{F}_{m} \tilde{\boldsymbol{x}}_{k-1}+\boldsymbol{G} \tilde{u}_{k}+\boldsymbol{w}_{k}$

where $\tilde{\boldsymbol{x}}=\boldsymbol{x}-\boldsymbol{x}_{m}$ is the state relative to the linearization point and $\tilde{u}=T_{\mathrm{e}}-T_{\mathrm{em}}$ is the engine torque difference from the equilibrium torque. The transition matrix is given by $\boldsymbol{F}_{m}=\boldsymbol{I}+\partial f /\left.\partial \boldsymbol{x}\right|_{\boldsymbol{x}_{m}, u_{m}} \Delta s$. Using the model from before $\boldsymbol{F}_{m}$ and $\boldsymbol{G}$ become

$\boldsymbol{F}_{m}=\left[\begin{array}{ccc}1+\frac{d v_{m}}{d s} \Delta s & 0 & -\frac{m g}{m_{\mathrm{t} m} v_{m}} \cos \alpha_{m} \Delta s \\ 0 & 1 & \cos \alpha_{m} \Delta s \\ 0 & 0 & 1\end{array}\right]$

$\boldsymbol{G}=\left[\begin{array}{c}\frac{i_{\mathrm{t} m} i_{\mathrm{f}} \eta_{\mathrm{t} m} \eta_{\mathrm{f} m}}{r_{\mathrm{w}} m_{\mathrm{t} m} v_{m}} \Delta s \\ 0 \\ 0\end{array}\right]$

where

$\frac{d v_{m}}{d s}=-\frac{i_{\mathrm{t} m} i_{\mathrm{f}} \eta_{\mathrm{t} m} \eta_{\mathrm{f} m}}{r_{\mathrm{w}} m_{\mathrm{t} m}} \frac{T_{\mathrm{e} m}}{v_{m}^{2}}-\frac{\frac{1}{2} c_{\mathrm{d}} A_{\mathrm{a}} \rho_{\mathrm{a}}}{m_{\mathrm{t} m}}+\frac{m g}{m_{\mathrm{t} m} v_{m}^{2}}\left(c_{r}+\sin \alpha_{m}\right)$

The equilibrium point $\boldsymbol{x}_{m}$ for the most common mode, cruising under engine power on the top gear, is obtained by choosing $v_{m}=80 \mathrm{~km} / \mathrm{h}, z_{m}=0 \mathrm{~m}, \alpha_{m}=0 \%$ grade, and the nominal vehicle specific values for $i_{\mathrm{t} m}, \eta_{\mathrm{t} m}$, and $\eta_{\mathrm{f} m}$. The driveline efficiencies and transmission gear ratio will also directly give $m_{\mathrm{t} m}$. As a result the input torque equilibrium is

$T_{\mathrm{e}_{m}}=\frac{\frac{1}{2} c_{\mathrm{d}} A_{\mathrm{a}} \rho_{\mathrm{a}} v_{m}^{2}+r_{\mathrm{w}} m g\left(c_{\mathrm{r}}+\sin \alpha_{m}\right)}{i_{\mathrm{t}_{m}} i_{\mathrm{f}} r_{\mathrm{w}} \eta_{\mathrm{t}_{m}} \eta_{\mathrm{f}_{m}}}$

for each mode of the linear system.

\subsection{Measurement equation}

Two measured states and the input signal engine torque, $T_{\mathrm{e}}$, are available for estimation of the system model state. The measured states are the vehicle velocity $v$ and the altitude $z$. This leads to a linear measurement equation

$\boldsymbol{y}_{k}=\underbrace{\left[\begin{array}{lll}1 & 0 & 0 \\ 0 & 1 & 0\end{array}\right]}_{\boldsymbol{H}_{k}} \underbrace{\left[\begin{array}{c}v_{k} \\ z_{k} \\ \alpha_{k}\end{array}\right]}_{\boldsymbol{x}_{k}}+\underbrace{\left[\begin{array}{c}e_{k}^{v} \\ e_{k}^{z}\end{array}\right]}_{\boldsymbol{e}_{k}}$

that is used with both the linear and non-linear vehicle models. The measurement noise for the two states is described by the white noise processes $e_{k}^{v}$ and $e_{k}^{z}$, respectively.

\section{Iterative road grade estimation}

Based on the model created in the previous section Kalman filtering is used to estimate states from measured data. Two different filters are investigated; an extended Kalman filter that uses the non-linear vehicle model, and a standard Kalman filter that is based on the linearized version of the vehicle model. Section 4.1 outlines the different steps of the road grade estimation. The sensors used to collect measurements are treated in Section 4.2. In Section 4.3 the Kalman filter used for state estimation and smoothing is described. This is followed by a discussion on fusion of estimation results from multiple measurements in Section 4.4. This is followed by a section summary that also includes a discussion on the chosen filtering approach. 


\subsection{State estimation}

Fig. 5 shows a schematic view of how the available sensor signals are used together with previously stored road grade information to generate an updated map. Three signals, the vehicle velocity $v$, the absolute altitude $z$, and the engine torque $T_{\mathrm{e}}$ are used directly with the model and Kalman filters to produce road grade estimates. The selected gear signal is used to choose appropriate values for the time-varying parameters in the system model. The Kalman filters also need the error covariance matrices $\boldsymbol{Q}$ and $\boldsymbol{R}$ to be set. These are adjusted based on the number of available satellites, if the vehicle is shifting gears, and whether the brakes are applied.

Once the Kalman filter has produced a complete system state trajectory estimate for a segment of the road, this segment is processed again in a smoothing step, in order to use all recorded sensor information for the estimation at each distance index. The smoothed grade and altitude estimates are then fused with any existing data for the road segment. Finally, the new road grade map segment is stored to the database.

\subsection{Sensors}

An important property of the presented road grade estimation method is that it only relies on sensors that are commonplace in HDVs. It is thus suitable for deployment in a large number of vehicles, without significant hardware costs, given that the computational and data storage requirements can be met by the vehicle platform. The restriction of creating a method based on existing mass market sensors limit the accuracy that can be expected from the input data. The grade estimator is thus designed to use many measurements from the same location in the map creation process.

The method relies on two continuous signals to be sensed in the vehicle driveline; the engine torque $T_{\mathrm{e}}$, and the vehicle speed $v$. Information is also required about the current gear, when gearshifts occur, and when any of the braking systems present in the vehicle are activated. Modern HDVs generally feature a distributed control system, with a number of interconnected electronic control units. These control units communicate in a network, usually an implementation of the controller area network (CAN) described in the ISO standard (11898-1:2003, 2003). The vehicles used in this study broadcast all the needed signals on their CAN buses.

The speed sensor is a part of the anti-locking brake system. The average of the sensed speed on the front wheels is used as the vehicle speed. The current gear and gearshift signals are broadcast by the gearbox control unit. The engine torque is calculated and broadcast by the engine control unit. It is determined by the engine state, and how much fuel is injected during each cycle.

In addition to the driveline signals the absolute position of the vehicle as derived from a GPS is also used. Satellite based positioning systems are rapidly becoming ubiquitous not only as personal navigation devices, but also built into many complex products. In the near future it is anticipated that the majority of HDVs being sold will have at least one satellite based positioning device built in. Access to the absolute vehicle position opens up the possibility of repeated measurement of a particular road segment using vehicle sensors. In this work that is utilized in order to update a road grade estimate each time the vehicle has passed over a certain road segment. A description of the GPS is beyond the scope of this work, but a good starting point for a general overview without too much mathematics is (El-Rabbany, 2006). A thorough treatment of the subject, including the relevant equations, is given in Misra and Enge (2006).

The accuracy of the position given by the GPS is very important for the correct functioning of the grade estimator. The error in the vertical position reported by the GPS will directly influence the estimated road grade, and the horizontal positioning error will cause grade estimates from different points on the road to be merged together. Since the proposed system is designed for use in a cost-sensitive environment a standard low-cost single channel GPS receiver will have to suffice. Such a receiver delivers what is called a standard positioning service, and has a typical $10 \mathrm{~m}$ horizontal and $15 \mathrm{~m}$ vertical accuracy. The accuracy figure is given as the 95th percentile of the error distribution (Misra \& Enge, 2006, p. 49).

GPS position estimates are generally bias free when averaged over long time periods and the error is approximately normally distributed. In the proposed grade estimation application this means that adjacent GPS measurement points will likely have a slow varying bias, with a period of several hours. Grade estimates for a specific location, however, will be based on uncorrelated normally distributed measurements. This is a prime motivation for developing a method for fusion of many measurements spread over time into one road grade estimate.

\subsection{Kalman filtering}

Two different Kalman filters are used to estimate the road grade and other model states. The non-linear vehicle model is used together with an extended Kalman filter (EKF), and the

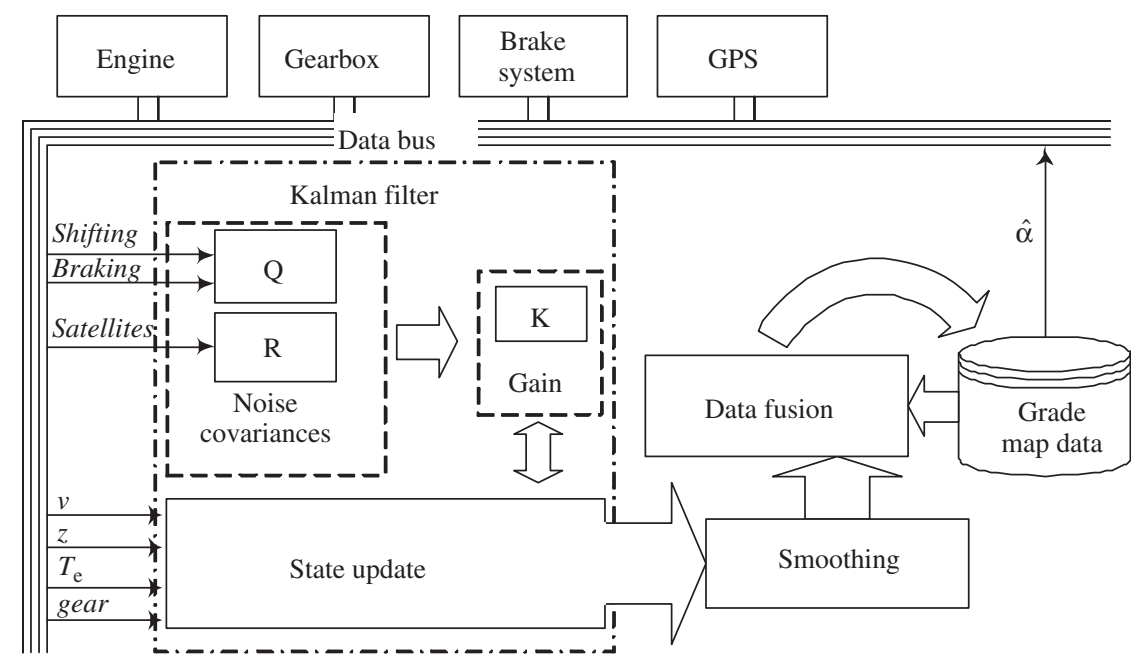

Fig. 5. Overview of the data filtering, smoothing and fusion of the proposed road grade estimation method. 
piecewise linear model with a standard Kalman filter (KF). Using the notation of the previous section, the system model to be used in the filtering with the EKF is given by

$\boldsymbol{x}_{k}=f\left(\boldsymbol{x}_{k-1}, u_{k}\right)+\boldsymbol{w}_{k}$

$\boldsymbol{y}_{k}=\boldsymbol{H} \boldsymbol{x}_{k}+\boldsymbol{e}_{k}$

In the EKF the non-linear model is linearized around the current state at every distance step. The obtained transition matrix $\boldsymbol{F}_{k}$ is then used to complete the steps of the standard Kalman filter recursions. These recursions are described by two update steps: a distance update, or prediction, step and a measurement update. In the distance update the system model is used to predict the future state of the system. Using the notation $\hat{\boldsymbol{x}}_{k \mid k-1}$ to denote the quantity $\hat{\boldsymbol{x}}$ at distance index $k$ based on information available up to distance index $k-1$ the distance update is done according to

$\hat{\boldsymbol{x}}_{k \mid k-1}=f\left(\hat{\boldsymbol{x}}_{k-1 \mid k-1}, u_{k}\right)$

$\boldsymbol{P}_{k \mid k-1}=\boldsymbol{F}_{k} \boldsymbol{P}_{k-1 \mid k-1} \boldsymbol{F}_{k}^{T}+\boldsymbol{Q}_{k}$

Similarly to $\boldsymbol{F}_{m}$ in the piecewise linear model the transition matrix $\boldsymbol{F}_{k}$ is defined to be the Jacobian $\boldsymbol{F}_{k}=(\partial f / \partial \boldsymbol{x})\left(\hat{\boldsymbol{x}}_{k-1 \mid k-1}, u_{k}\right) . \boldsymbol{P}_{k \mid k-1}$ is the estimated error covariance, and $\boldsymbol{Q}_{k}=E\left[\boldsymbol{w}_{k}^{2}\right]$ is the process noise covariance. After the distance update the measurement at distance index $k$ is used in a measurement update to improve the estimate. The measurement update is described by

$\boldsymbol{K}_{k}=\boldsymbol{P}_{k \mid k-1} \boldsymbol{H}^{T}\left(\boldsymbol{H P}_{k \mid k-1} \boldsymbol{H}^{T}+\boldsymbol{R}_{k}\right)^{-1}$

$\hat{\boldsymbol{x}}_{k \mid k}=\hat{\boldsymbol{x}}_{k \mid k-1}+\boldsymbol{K}_{k}\left(\boldsymbol{y}_{k}-\boldsymbol{H} \hat{\boldsymbol{x}}_{k \mid k-1}\right)$

$\boldsymbol{P}_{k \mid k}=\left(\boldsymbol{I}-\boldsymbol{K}_{k} \boldsymbol{H}\right) \boldsymbol{P}_{k \mid k-1}$

Here $\boldsymbol{K}_{k}$ is the Kalman gain, and $\boldsymbol{R}_{k}=E\left[\boldsymbol{e}_{k}^{2}\right]$ is the measurement noise covariance.

The piecewise constant linear model is used with a standard Kalman filter. At each mode change between different linearizations of the model the final state of the old filter is used to initialize the new filter.

One of the main challenges in using the grade estimation method on real data is that the true noise covariances $\boldsymbol{Q}$ and $\boldsymbol{R}$ are not known. A number of methods have been proposed for estimating these matrices, for example by Mehra (1970). In the proposed method the covariance matrices are used as design variables to tune the grade estimation filter to generate an accurate and reliable estimate. The matrices were adjusted until the estimation error for a training set of measurement data was sufficiently small.

To simplify the choice of these design parameters the noise covariance matrices were chosen to be diagonal. For the measurement noise this seems reasonable since the vehicle speed and GPS altitude are obtained through independent sensors measuring different quantities, whose measurement error can be assumed independent. For the process noise the situation is more complicated. The random walk road grade model used will lead to unmodeled systematic changes in the road grade. These changes will also affect the altitude. It is likely that such an error would have an effect on the velocity error as well, since the road grade used in that calculation is faulty. The magnitudes of these effects are hard to estimate, since they depend on the magnitudes of the model errors themselves. In this version of the method the results when using a diagonal $\boldsymbol{Q}$ matrix are investigated, evaluation of possible improvements with a full matrix are left as future work. This simplification is known to somewhat increase the error in the final estimates.

\subsubsection{Smoothing}

Filtering introduces filter delay in the estimates. Since the aim of the proposed method is to estimate the road grade at a specific position along the road it is important to compensate for this delay. By carrying out the road grade estimation off-line, when a complete road segment has already been recorded, the filtering delay can be removed through a process commonly referred to as smoothing. An added benefit is that after smoothing all available estimation data, both before and after the current position is used in the estimate for each data point. The Rauch-Tung-Striebel fixed point smoothing algorithm, introduced in Rauch, Tung, and Striebel (1965), yields optimal smoothed estimates and is suitable for use together with Kalman filtering. It is used to find $\hat{\boldsymbol{x}}_{k \mid N}$ where $N$ is the total number of data points collected during one run along the road segment. In practice the smoothing is performed backwards along the road, and uses quantities generated by the Kalman filter.

The predicted quantities at the last position of the road segment, where $k=N$, are used to initialize the recursion. This gives access to $\boldsymbol{P}_{N+1 \mid N}$ and $\hat{\boldsymbol{x}}_{N+1 \mid N}$ in the first step of the smoothing recursion. The superscript $s$ is used to indicate smoothed quantities. $\boldsymbol{P}_{k}^{s}$ denotes the smoothed error covariance, $\hat{\boldsymbol{x}}_{k}^{s}$ is the smoothed state estimate, and $\boldsymbol{K}_{k}^{s}$ is the smoothing gain. The smoothing backwards recursion is given by

$$
\begin{aligned}
& \boldsymbol{K}_{k}^{s}=\boldsymbol{P}_{k \mid k} \boldsymbol{F}_{k}^{T} \boldsymbol{P}_{k+1 \mid k}^{-1} \\
& \hat{\boldsymbol{x}}_{k \mid N}^{s}=\hat{\boldsymbol{x}}_{k \mid k}+\boldsymbol{K}_{k}^{s}\left(\hat{\boldsymbol{x}}_{k+1 \mid N}^{s}-\hat{\boldsymbol{x}}_{k+1 \mid k}\right) \\
& \boldsymbol{P}_{k \mid N}^{s}=\boldsymbol{P}_{k \mid k}+\boldsymbol{K}_{k}^{s}\left(\boldsymbol{P}_{k+1 \mid N}^{s}-\boldsymbol{P}_{k+1 \mid k}\right) \boldsymbol{K}_{k}^{S} T
\end{aligned}
$$

\subsection{Data fusion}

When a quantity is measured more than once both the confidence in the measurement and its precision are generally increased. With multiple estimates statistical properties of the estimation scheme can be studied. Given that the estimation method is unbiased an increasing number of estimates will give a better map. The effects of temporary disturbances such as local loss of the GPS signal and braking are also better handled when data from multiple runs are used.

In order to merge data from many runs along the same road segment a distributed data fusion method is used. The distributed approach has the important advantage that the amount of road data that have to be stored does not increase as additional estimates from known road segments are incorporated into the map. For each road segment, the map consists of the road related states (altitude $z$ and grade $\alpha$ ) and the associated estimated error covariance estimates for those states. The vehicle velocity is not of any interest in the map, since it is not a road property. Based on the estimated error covariances stored in the map and the estimated error covariances of a new smoothed estimate an updated map is created each time a new estimate of a road segment becomes available.

Assuming that the errors in estimates from each run along the road are entirely uncorrelated, the quantities for the new map can be calculated as follows:

$\boldsymbol{P}_{k}^{f}=\left(\left(\boldsymbol{P}_{k}^{1}\right)^{-1}+\left(\boldsymbol{P}_{k}^{2}\right)^{-1}\right)^{-1}$

$\hat{\boldsymbol{x}}_{k}^{f}=\boldsymbol{P}_{k}^{f}\left(\left(\boldsymbol{P}_{k}^{1}\right)^{-1} \hat{\boldsymbol{x}}_{k}^{1}+\left(\boldsymbol{P}_{k}^{2}\right)^{-1} \hat{\boldsymbol{x}}_{k}^{2}\right)$

where $\boldsymbol{P}_{k}^{f}$ is the resulting error covariance, $\hat{\boldsymbol{x}}_{k}^{f}$ is the new state estimate for the map. The quantities $\boldsymbol{P}_{k}^{1}, \boldsymbol{P}_{k}^{2}, \hat{\boldsymbol{x}}_{k}^{1}$, and $\hat{\boldsymbol{x}}_{k}^{2}$ are the source estimates and estimated error covariances. The superscripts 1 and 2 refer to the two input data sets, one being the 
current map and the other containing estimates from the run being processed. The superscript $f$ refers to the output fused estimate. This data fusion method is described in more detail in e.g. Gustafsson (2000).

The assumption in (23) that estimation errors in the two source samples are uncorrelated is somewhat troublesome. Since the estimates are based on repeated measurements using the same method and road, it is unlikely that this is fully satisfied. A main source of correlation is the difference between the road model (a random walk) and the true road. An important area of further study is if the results can be improved by using a more advanced road model. Not accounting for the correlated process noise through the use of a diagonal $\mathbf{Q}$ matrix in the estimation, as described in Section 4.3, also increases correlation in the estimation errors. The correlation will lead to an underestimate of the state error covariances in $\boldsymbol{P}_{k}^{f}$. Over time new estimates will start having little influence on the already stored data.

Another important caveat is that in practice covariance terms between the altitude and road grade states, represented by the off-diagonal terms of the source matrices $\boldsymbol{P}_{k}^{1}$ and $\boldsymbol{P}_{k}^{2}$ actually degrade the merged result. Uncertainties in the estimation of these quantities in the Kalman filtering and smoothing steps occasionally cause the weighting factors to give a combined estimate that is not in the interval $\left[\hat{\boldsymbol{x}}_{k}^{1}, \hat{\boldsymbol{x}}_{k}^{2}\right]$. Currently this problem is solved by only using the diagonal elements of $\boldsymbol{P}_{k}^{1}$ and $\boldsymbol{P}_{k}^{2}$ in the fusion. The result is that only the estimated covariance of each of the states will affect how much weight the measurement of that state has in the merge, the estimated cross correlation between altitude and road grade errors will be ignored.

\subsection{Summary}

A road grade estimator based on standard HDV sensors has been developed on the form shown in Fig. 5. The estimator uses a Kalman filter where the noise and process variances are adapted to driving events. Smoothing is used to compensate for filtering delay. The estimator produces spatially sampled estimates that are fused together with the aggregate of previous estimates of the road grade at that location.

The Kalman filter in the estimator is known to produce an optimal state estimate in the minimum mean square error sense, given that the system is linear and that the measurement and process noises are truly white, Gaussian, and have the assumed covariances. Since the studied system is non-linear, with model and sensor errors that most likely yield somewhat colored process and sensor noises, there is no proof neither that the estimates will be optimal, nor that they will even converge. The RTS smoothing similarly provides minimum mean square error estimates of the state at each position based on all the collected measurements, for systems that fulfill the same assumptions as for the Kalman filter. Since the EKF results are used as input to the smoothing recursion there is no proof that the output will be optimal, or that it will converge. Using the linearized model only moves the noncompliance with assumptions about the model to the assumptions about the noises. In this case not only the previously existing model errors, but also linearization errors contribute to coloring the noise.

Similarly, optimal estimation based on all available experiments would yield a very large Kalman filter, including two states to describe the road, and one velocity state for the vehicle used in each experiment. By only fusing the road states based on their estimated error covariances, the problem of including models for all the vehicles in the filter is avoided. As a consequence the estimate will no longer be optimal. The applied fusion method includes the assumption that the different experiments observe the system with different realizations of the process noise. This is not strictly true, the road realization is the same each time the vehicle passes over it. When the process noise is correlated between experiments, the distributed fusion method will underestimate the error covariance matrix. As noted in Section 4.4 issues with the cross-correlation between the altitude and slope states further moves the implemented method away from the theoretical optimum.

\section{Experiments}

The proposed road grade estimation algorithm has been tested on experimental data collected using HDVs driving on a Swedish highway. The road tests verify the applicability of the method, but also provide insights on areas of possible future improvements. This section describes the experiments that have been made and the obtained results.

\subsection{Experimental setup}

Road tests with the proposed grade estimation algorithm have been carried out on a part of highway E4 from Södertälje to Nyköping, as shown in Fig. 6. The test road contains a mix of hills with road grades between $-4 \%$ and $4 \%$ and flat segments. This gives an opportunity to study how the estimation algorithm handles various highway driving situations. To illustrate details in the behavior of the road grade estimation algorithm results are presented for the full $29 \mathrm{~km}$ long southbound, and $38 \mathrm{~km}$ long northbound road segments, as well as a short segment where the vehicle is braking during some of the experiments. In order to effectively evaluate the obtained road grade estimates a reference grade profile is required. The reference profile used in this work has been obtained from a high-quality 3D trajectory measurement system based on a tightly coupled GPS and inertial navigation system (Oxford Technical Solutions Limited, 2008).

The configuration and important parameters of a HDV can vary substantially, therefore three different vehicles have been used to verify the applicability of the grade estimation method in each

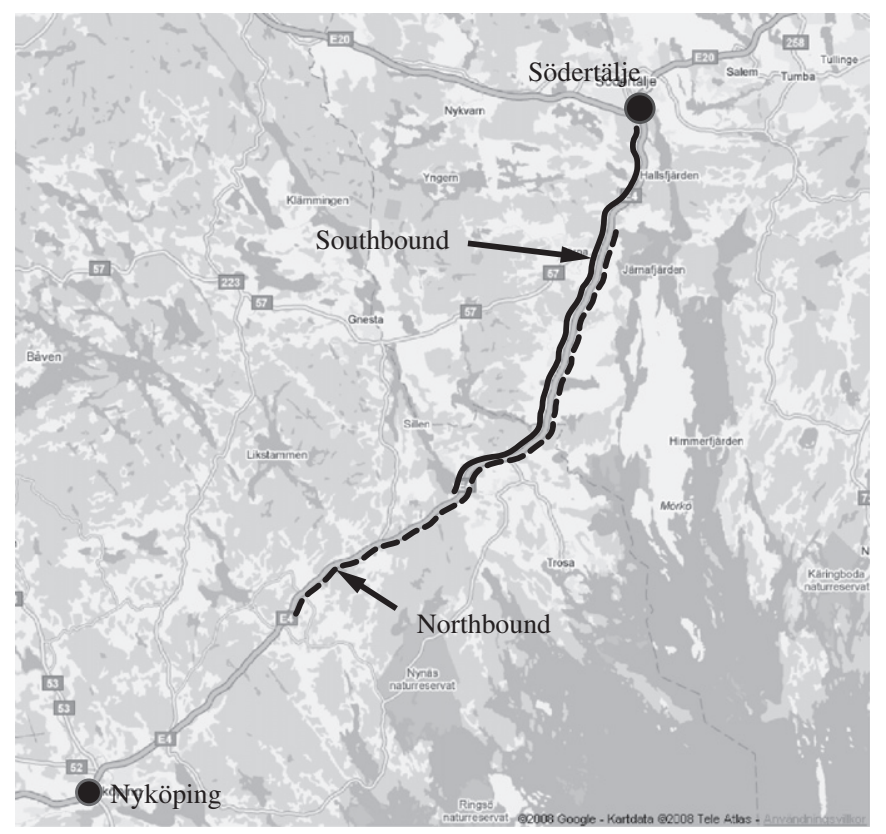

Fig. 6. Map of the test area. Experiments have been conducted on highway E4 between Södertälje and Nyköping. (Image courtesy of Google.) 
case. The types of test vehicles used are illustrated in Fig. 2. The test vehicles have weights ranging from 12 to $39 t$ and between 2 and 5 axles. Important properties for the test vehicles are listed in Table 1. The modeled external forces described in Section 3.1 depend heavily on the vehicle parameters used in the equations. Getting reasonable values for these can be a challenge. In this work the vehicle parameters have been chosen based on information about the vehicle configurations, but without calibration for the individuals. The total vehicle weight in each experiment is assumed to be known in the road grade estimator, so it has been measured using a scale.

Experimental data were collected while the test vehicles were being driven at normal cruising speed along the highway. A laptop computer with a controller area network (CAN) bus interface card was used to log both vehicle signals and GPS data. The scenario is illustrated in Fig. 7.

\subsection{Experimental results}

The proposed grade estimation algorithm shows promising results. The estimation has been carried out using the non-linear vehicle model and EKF, at the end of Section 5.2 a comparison is made with the linear model and KF. On the southbound test road as a whole the root mean square error (RMSE), defined as

$R M S E=\sqrt{E\left((\hat{\alpha}-\alpha)^{2}\right)}$

of the estimated road grade was $0.16 \%$ grade. A relatively large part of the error was due to a bias of $-0.08 \%$ grade. For the northbound direction the RMSE was $0.18 \%$ grade, with a bias of $-0.12 \%$ grade. Even if individual measurements sometimes are far from the true road grade, the merged estimate from a few runs along the road comes quite close over most of the distance, the estimation results for the southbound direction are shown in Fig. 8. Events such as braking and shifting generally decreases the quality of the road grade estimate for that experiment, but most of the time they not occur at the exact same position in all experiments. As a result of the data fusion many segments with lower quality data can be identified and suppressed in the final estimate. The main performance criterion used to evaluate results in this study is the RMSE of the grade estimate compared to the

Table 1

Key properties and specifications for the test vehicles used to collect experimental data.

\begin{tabular}{llllr}
\hline Vehicle & Configuration & Weight $(\mathrm{t})$ & Axles & Exp. \\
\hline A & Tractor and semi-trailer & 39 & 5 & $1,2,3$ \\
B & Tractor & 12 & 2 & 4,5 \\
C & Rigid truck & 21 & 3 & 6 \\
\hline
\end{tabular}

The total vehicle weight is given in tons.

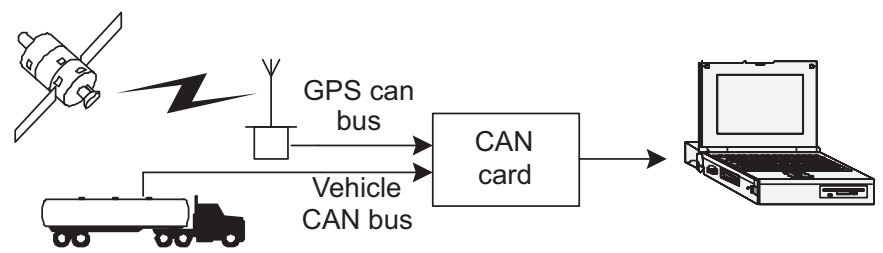

Fig. 7. During the road tests measurement data were collected using a laptop computer with a dual channel CAN interface card. Vehicle data were logged through one of the channels, and the other one was used to collect GPS messages.

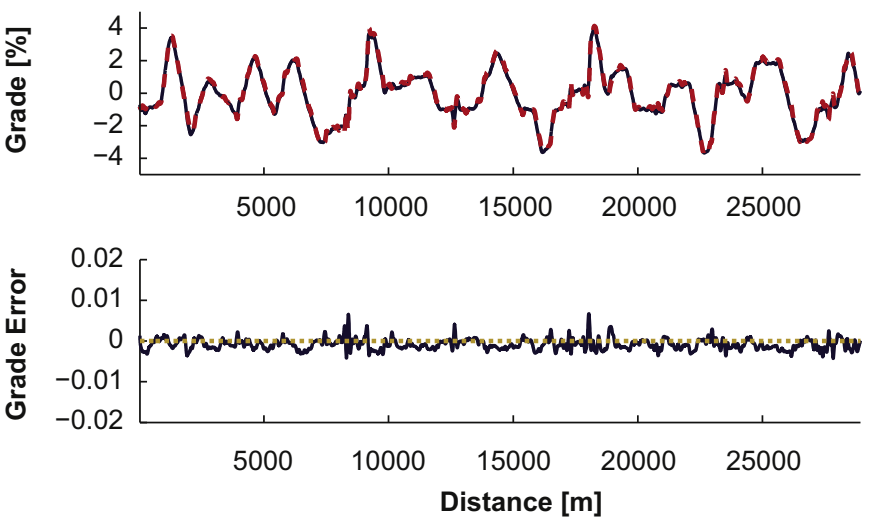

Fig. 8. The first part shows the estimated road grade profile for the southbound direction on highway E4 south of Södertälje, based on six road experiments with three different vehicles (solid), compared to a reference road grade profile (dashed). The second part shows the error in the estimated grade.

reference road grade, as defined in Eq. (24), expressed as percent grade.

\subsubsection{Sensor data}

Example sensor data recorded on a $12 \mathrm{~km}$ segment of the southbound test road are used to illustrate the different input signals used by the state estimator. For clarity the figures only contain data from one run along the road for each of the three test vehicles. The ones chosen are experiments two (solid line, vehicle A), four (dashed line, vehicle B) and six (dotted line, vehicle C). Fig. 9 shows the recorded sensor signals used in the estimator.

Vehicle A is heavy in relation to its engine power, and has a typical speed profile for an economically driven HDV. The driver has ordered the speed to be lowered ahead of long downhill segments, thus reducing the amount of braking necessary. Vehicle $B$ is very light, more powerful than vehicle $A$, and was operated with a constant cruise control system enabled. The speed profile is very flat with no speed loss in uphill segments, and no run-out in downhill segments. Vehicle $C$ is heavy enough to gain some extra speed in downhill segment, but was following slower moving traffic for part of the drive. At 18-19 km of experiment 6 with vehicle $C$ there were roadworks in one of the lanes, with an associated mandated speed decrease. The effects of loss of satellite coverage can be seen clearly around $24-26 \mathrm{~km}$ in the altitude trace for vehicle $B$, but short signal loss also occurs with vehicles $A$ and $C$. It is interesting to note the large almost static difference between the altitudes reported by vehicles $\mathrm{B}$ and $\mathrm{C}$, and the one from vehicle $A$. This difference of approximately $10 \mathrm{~m}$ is by no means unusual for the kind of GPS system used, i.e. without differential corrections applied. The key advantages of the GPS altitude signal is that the error changes very slowly, and that its mean is zero when taken over very long time periods.

It can be seen in the figure that vehicle A uses its maximum power for long periods of time in the uphill segment, and applies no engine power in a number of downhill segments. The very light vehicle $B$ never utilizes the full engine power, and rarely has a zero utilization. Vehicle $C$ has to work slightly harder on average than vehicle $\mathrm{B}$, but also shows a number of gear changes and peak power utilizations during acceleration. Gear changes can be seen in the torque data as short spikes towards zero.

It is important to know when any of the vehicles' brakes are applied, since this leads to an external, unknown, torque entering the equations of motion. The "braking" signal indicates when the 

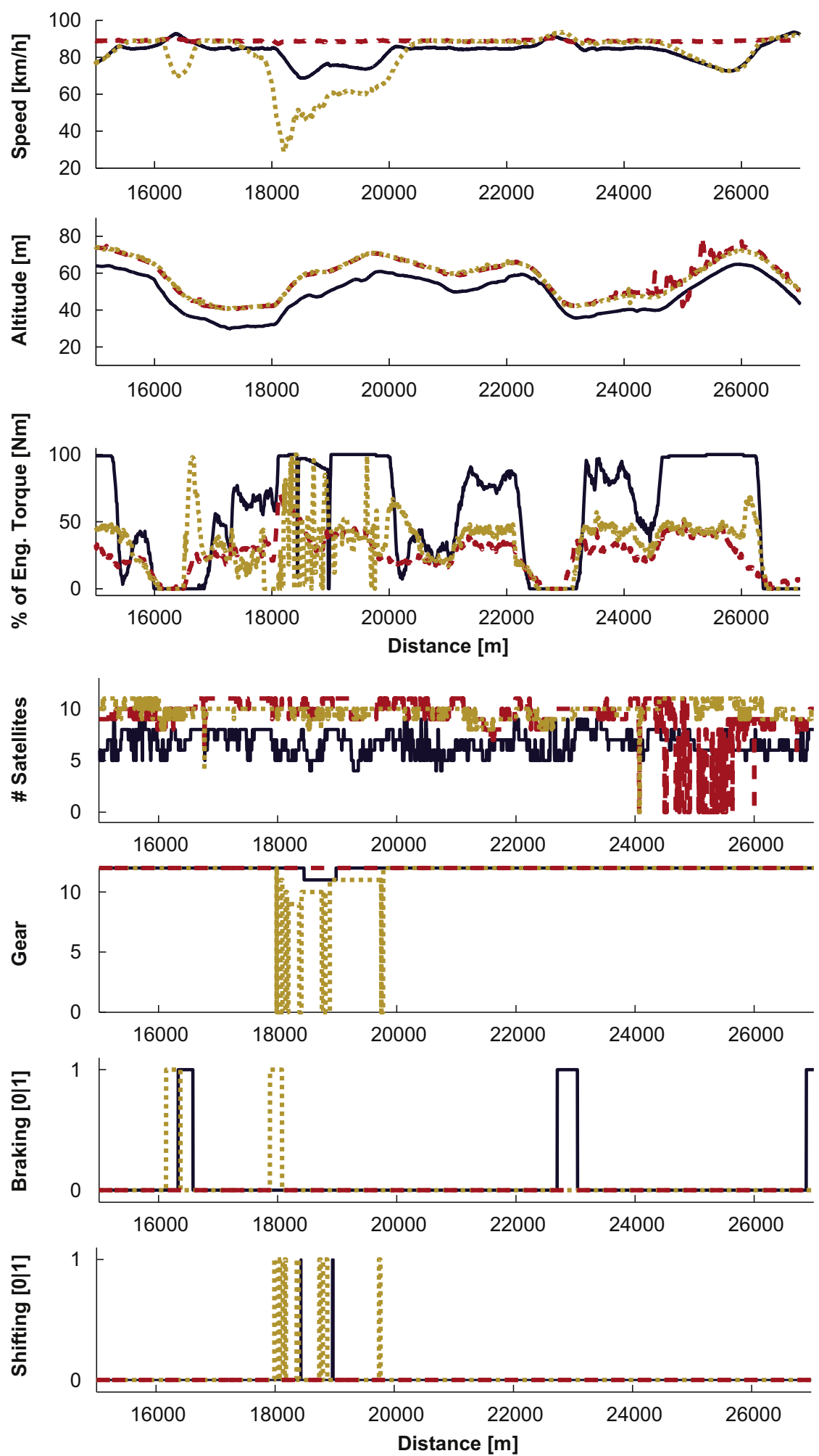

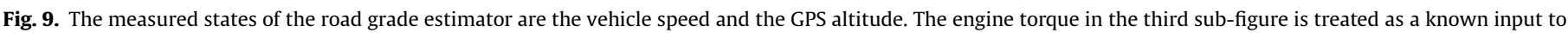

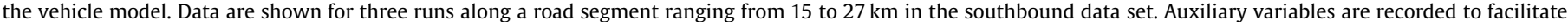

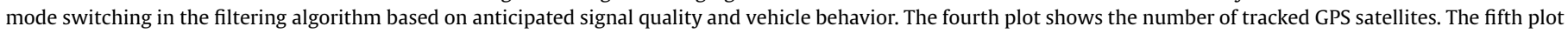

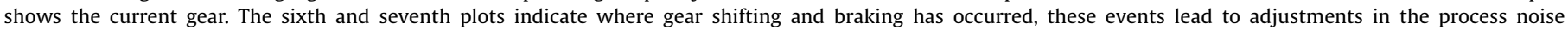
assumptions. The solid line represents experiment 2 (vehicle A), the dashed line is experiment 4 (vehicle B) and the dotted line is experiment 6 (vehicle C).

longitudinal vehicle model should not be trusted for this reason. Vehicle A applies the brakes to avoid overspeeding, while vehicle $C$ uses them to avoid running into traffic ahead.

\subsubsection{Braking}

One of the most challenging situations for the grade estimator is when the vehicle applies one of the brake systems. Therefore, 
one such occasion is described in more detail. During braking the engine will generally report a negative net torque from internal friction when fueling is cut off. This means that the vehicle model based prediction in the grade estimator will be computed using only the engine friction as its driveline input, even though there is a braking force present as well. The roll resistance and air drag will still be correctly modeled. The missing brake force will then be attributed to the gravity component, i.e. the road grade. This should lead to a road grade estimate that is too high (more uphill than in real life). In order to avoid this effect, and at the same time raise a flag to the data fusion algorithm that these data deserve lower trust than the norm, the process noise covariance for the velocity state is increased. This causes the estimator to rely less on the vehicle model, and more on past road grade estimates and the GPS measurements. At the same time the estimated error covariance of the output increases.

The example segment shown in Fig. 10 represents a comparatively steep downhill segment, where the heavy vehicle
A needs to apply the brakes to avoid exceeding its set maximum allowed speed. Data are shown for experiments 2 (solid, vehicle A), 3 (dashed, vehicle A), and 6 (dotted, vehicle C). Vehicle C does not apply the brakes in this segment. Fig. 10 shows the key signals of interest during braking. The first two sub-figures show the velocity profiles and GPS altitude measurements, after that comes the reported engine torque and braking signal. Braking is required by vehicle $A$ for a total of almost $1 \mathrm{~km}$ in each of the experiments. The resulting road grade error in the fifth part of the figure shows that experiment 2 yields an overestimate of the grade during the first brake application, an underestimate during the second, and no visible additional error during the third application. Experiment 3 yields no significant additional error during the first application, and overestimates of the grade during the second. The final part of the figure shows the estimated grade error covariance after the smoothing step in the grade estimator. The estimated error variance almost doubles during the segments where the brakes are applied.
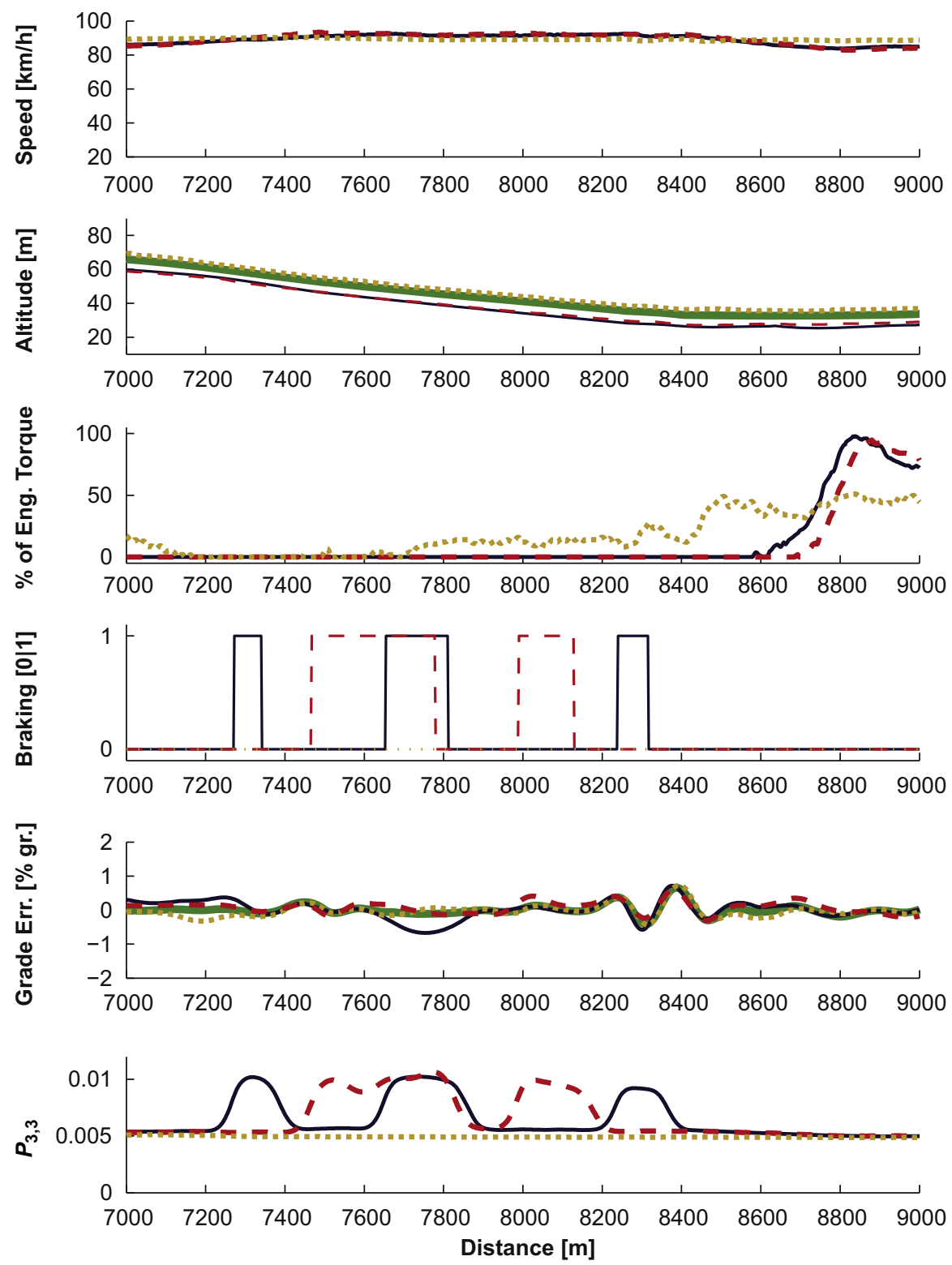

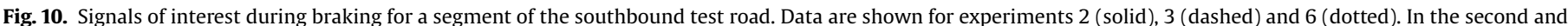
fifth parts the merged final estimate is shown as well (thick solid). 


\subsubsection{Iterative data fusion}

Using data from more than one run along the road and more than one vehicle improve the reliability of the final grade estimate. The downhill segment from $s=22150$ to $23150 \mathrm{~m}$ is one of the hardest parts of the test road to estimate accurately, it is therefore used as an example of how the data fusion step improves the quality of the final grade estimate. The grade maps resulting from the progressive inclusion of data from the six experiments can be seen in Fig. 11. As more data are added the road grade map is improved. The mean value of all included grade estimates at each sample point is also shown, to highlight the effect of the data fusion step. Each figure shows the latest experiment (dashed), the road grade map based on all experiments added so far (solid) and the reference road grade (dotted). a
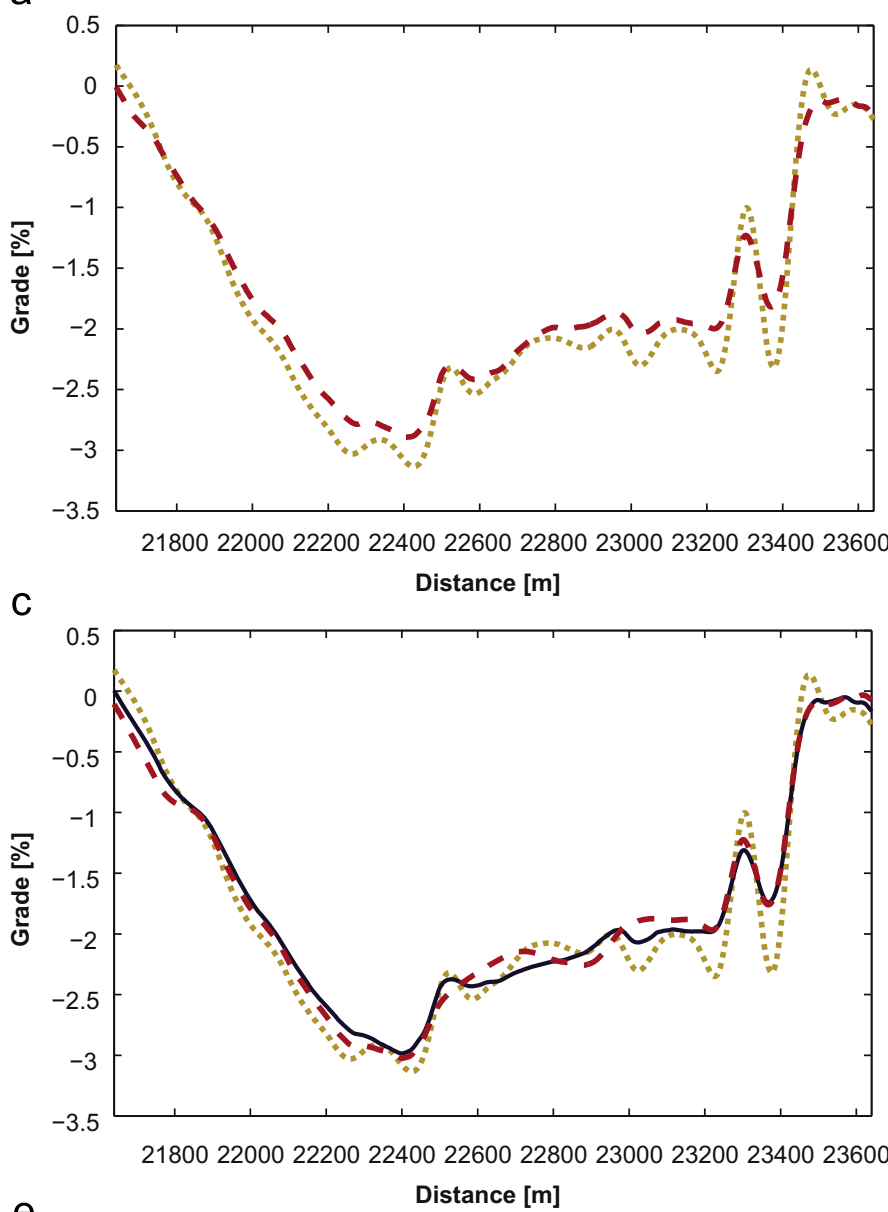

e

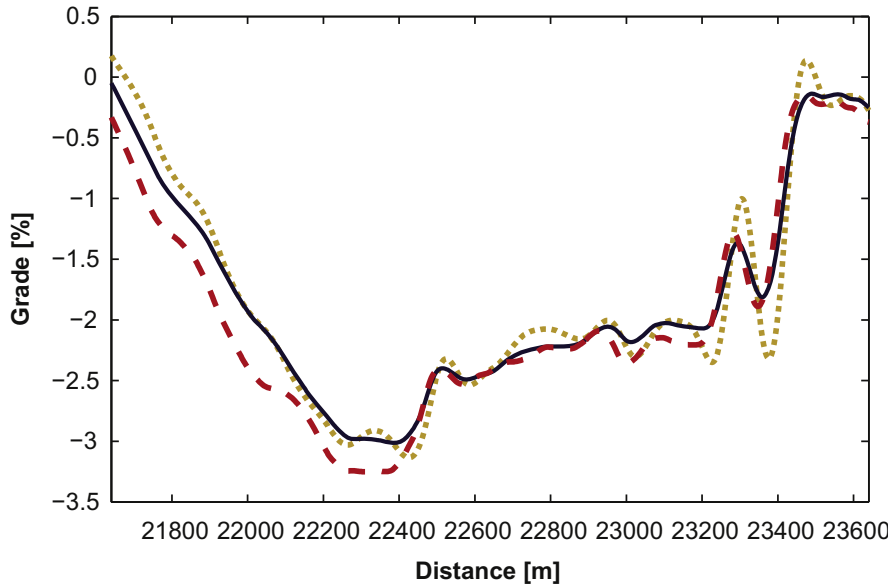

b
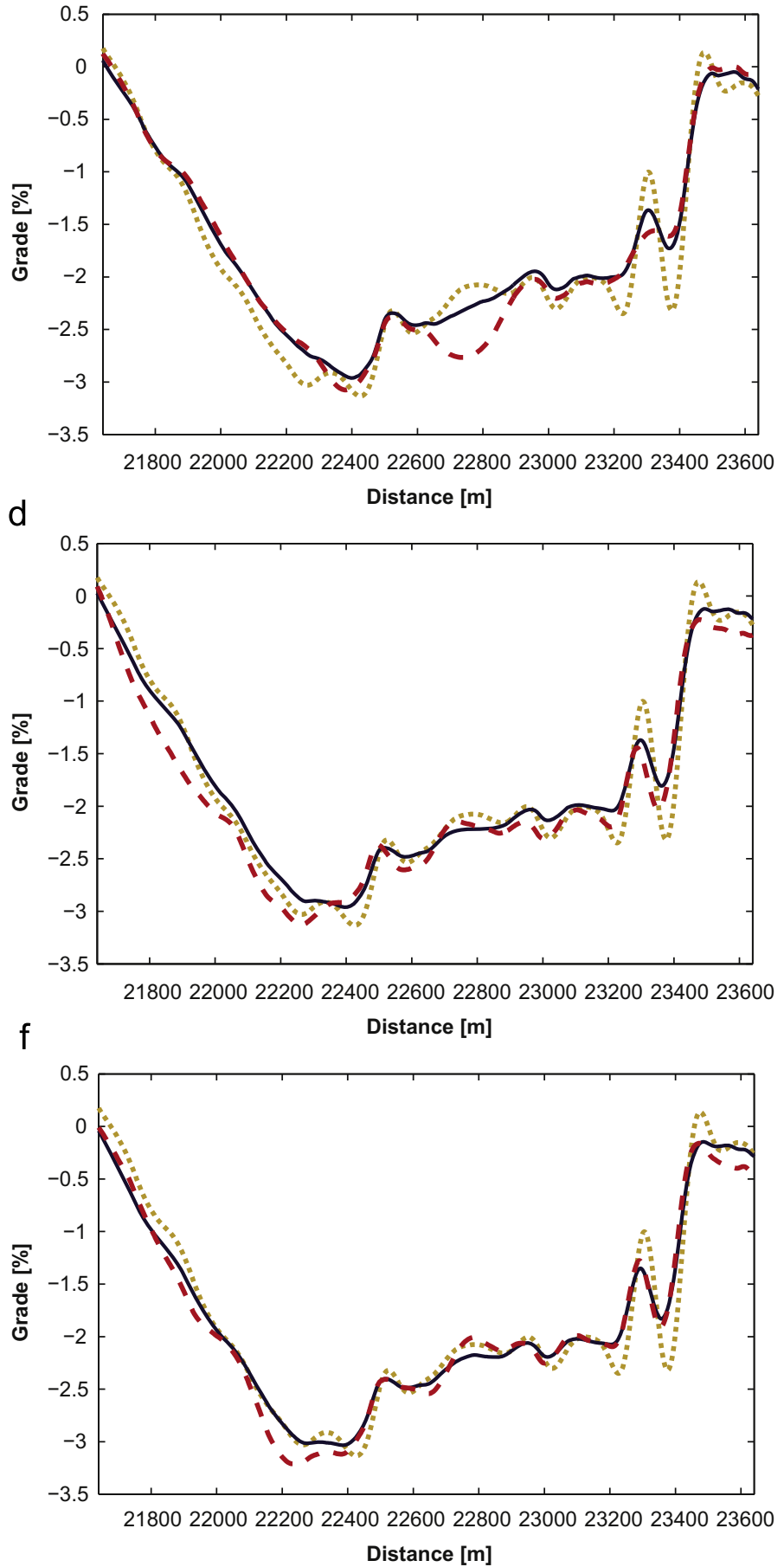

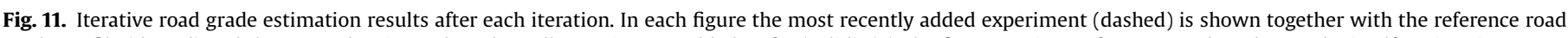

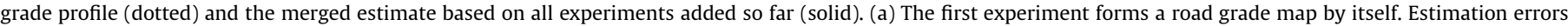

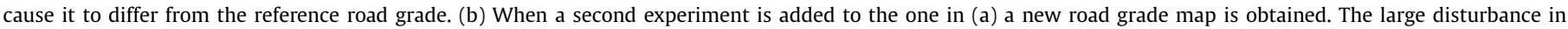

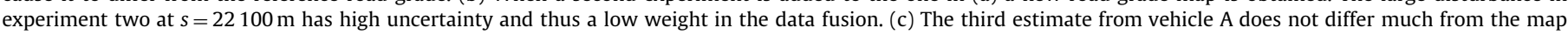

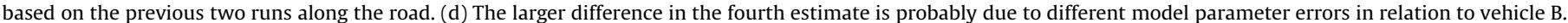
(e) Estimate five is based on vehicle B, just like the one in (d). (f) When the sixth estimate, recorded with vehicle C, has been added the map is complete. 


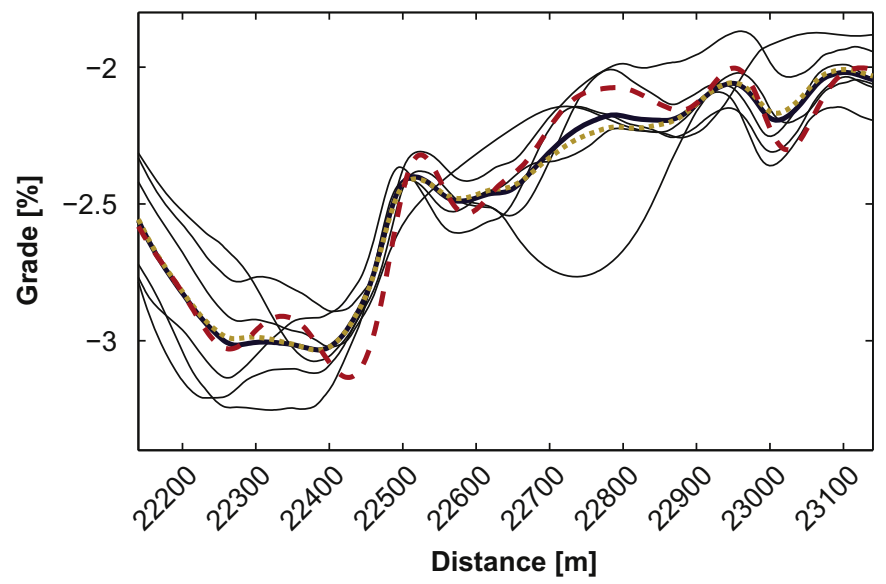

Fig. 12. The final merged road grade estimate (solid) is shown with the reference grade profile (dashed) and the mean value of all smoothed estimates (dotted). The estimates from the individual experiments are also included (thin lines). This is a magnification of the most challenging part of the test road. The estimate based on experiment two is particularly at odds with the rest at $22750 \mathrm{~m}$. This is due to a combination of poor satellite coverage and the effect of the braking. The detrimental effect on the fused estimate is smaller than on the mean.
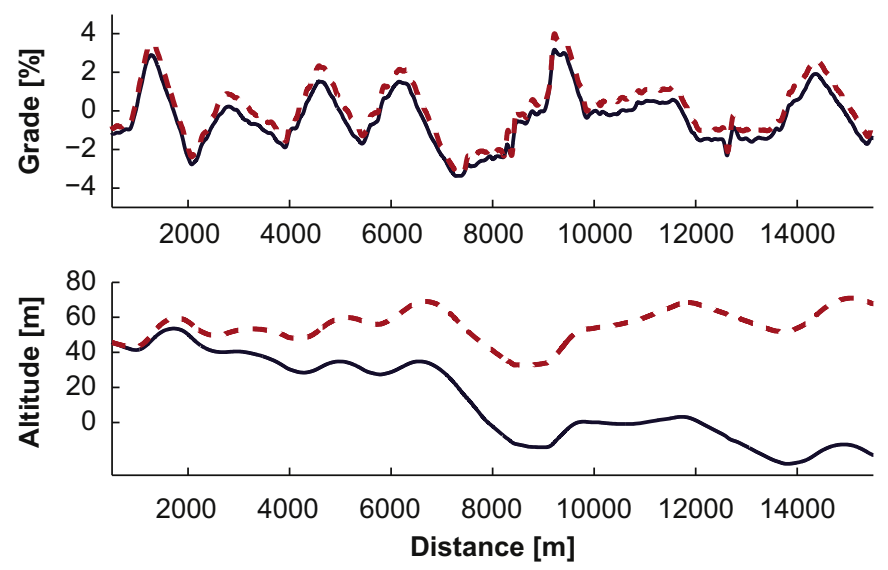

Fig. 13. The top part of the figure shows the estimated road grade without the GPS input (solid). This signal lies significantly below the reference profile (dashed). As a result the altitude estimate has a significant drift. Since there is no absolute altitude measurement available in the filter it is initialize to the same starting value as the reference profile.

Fig. 12 shows a comparison of the smoothed estimates from all six southbound experiments with the final grade estimate and the reference grade profile.

\subsubsection{Vehicle model bias and GPS altitude noise}

Without the GPS altitude measurements the vehicle model and measured signals give an estimated grade that has a bias due to modeling errors. The bias is reduced when the GPS altitude measurement is introduced as a vehicle independent low frequency correction in the filter. Depending on the vehicle parameters the magnitude of the drift varies. Fig. 13 shows the estimated grade and altitude when the grade estimator has been operated without GPS input data. Table 2 shows the mean grade biases observed in each of the experiments when compared with the road grade reference. The grade biases range from negligible for vehicle $A$ (experiments $1-3$ ), when using the GPS, to severe for vehicle B (experiments 4-5). It is evident that results could be improved if the parameters for vehicles $\mathrm{B}$ and $\mathrm{C}$ were better calibrated to match the vehicles and environmental conditions they are supposed to describe.
Table 2

Observed mean bias in estimated road grade, with and without GPS input, for the south- and northbound test roads.

\begin{tabular}{lllll}
\hline Dir. & Meas. & Vehicle & Mean bias w. GPS & Mean bias w/o GPS \\
\hline South & 1 & A & -0.02 & -0.15 \\
South & 2 & A & -0.04 & -0.18 \\
South & 3 & A & -0.03 & -0.34 \\
South & 4 & B & -0.12 & -0.88 \\
South & 5 & B & -0.18 & -1.28 \\
South & 6 & C & -0.09 & -0.58 \\
South & Merged & & -0.08 & -0.57 \\
North & 1 & A & -0.09 & -0.54 \\
North & 2 & A & -0.10 & -0.58 \\
North & 3 & A & -0.06 & -0.57 \\
North & 4 & B & -0.17 & -1.31 \\
North & 5 & B & -0.17 & -1.30 \\
North & Merged & & -0.12 & -0.86 \\
\hline
\end{tabular}

The road grade figures are given in percent.

While the GPS altitude signal is important for the overall estimation it is by itself not sufficient for a good grade estimate within a reasonable number of passes over the road. The signal is locally very noisy, and there are road sections that have no satellite coverage, e.g. tunnels.

\subsubsection{Linear system model}

The results from using the piecewise constant linear model instead of the time-varying non-linear model indicated only marginal changes in the estimated road grade. The main nonlinearity in the vehicle model, for the magnitude of road grades considered, is in the speed. The linear model is only valid for velocities close to the linearization point of $80 \mathrm{~km} / \mathrm{h}$. During most of the experiments the speed of the measuring vehicle was close to this value. The proposed method is primarily suited for highway estimation, and it would probably be wise to reject any data sets with large speed deviations, regardless of whether the linear or non-linear model is used.

\subsection{Summary}

Experimental data have been collected in a total of eleven experiments using three HDVs on a Swedish freeway. The six data sets from the southbound segment and the five from the northbound segment have been merged into two road grade maps. Overall the performance of the proposed method is promising, even though vehicle model or parameter errors cause a slight bias in the estimated grade. The estimation error increases during braking, but this is handled quite well in the data fusion. It has been noted that the GPS receiver is vital to bias rejection. Grade estimates based on the linearized model showed comparable results to those obtained with the nonlinear model.

\section{Conclusions}

Recent advances in the development of embedded electronics and low cost global positioning enable new vehicle control systems that use map data to supplement traditional sensors in building a model of the surroundings. These systems rely on digital maps that include accurate road geometry information. The road grade is an attribute of particular importance to many HDVs, and the required road grade maps are not currently at hand. A system model has been developed that links the road grade to sensor signals that are generally already available in the control network of vehicles. Using this system model an iterative method for creating and improving road grade maps has been developed. 
The method uses information about gear shifts, vehicle braking and satellite coverage to adapt the grade estimator and weight the influence of new grade estimates being added to the map.

The proposed method for grade estimation combines the long term stability of the GPS derived altitude signal with the short term smoothing and dead reckoning capabilities of the vehicle model based grade estimation. The inclusion of the GPS receiver counteracts much of the bias in the grade estimate introduced by errors in the vehicle model and its parameters. On the other hand the vehicle model is able to capture short term variations in the road grade much better than what would be possible using only the noisy derivative of the GPS receiver altitude signal.

Experiments have shown that already at this stage the proposed method is feasible for collecting road grade with an average RMSE of $0.17 \%$ grade for the two test roads. Previous practical experience, as well as a theoretical analysis, shows that road grade estimates of the obtained quality are useable for example in a look-ahead cruise controller based on road grade data.

\section{Acknowledgments}

This work is partially supported by Scania CV AB, the Swedish Program Board for Automotive Research (IVSS), the European Commission through the Network of Excellence HYCON, and the Swedish Research Council.

\section{References}

11898-1:2003, I. (2003). Road vehicles_controller area network (CAN)—part 1: Data link layer and physical signalling. ISO, Geneva, Switzerland.

Bae, H. S., Ruy, J., \& Gerdes, J. (2001). Road grade and vehicle parameter estimation for longitudinal control using GPS. In Proceedings of IEEE conference on intelligent transportation systems, San Francisco, CA.

Brüntrup, R., Edelkamp, S., Jabbar, S., \& Scholz, B. (2005). Incremental map generation with GPS traces. In Proceedings of IEEE intelligent transportation systems, Vienna, Austria.

Carlsson, A., Baumann, G., \& Reuss, H. C. (2008). Implementation of a self-learning route memory as an electronic co-driver for reduced emissions. In FISITA world automotive congress, Munich, Germany.

El-Rabbany, A. (2006). Introduction to GPS the global positioning system (2nd ed.). Boston: Artech House.

Fathy, H. K., Kang, D., \& Stein, J. L. (2008). Online vehicle mass estimation using recursive least squares and supervisory data extraction. In Proceedings of American control conference, Seattle, Washington, USA.

Fröberg, A. (2008). Efficient simulation and optimal control for vehicle propulsion. Ph.D. thesis, Linköpings universitet, May.
Gaeke, E. G. (1973). Road grade sensor. US Patent \#3,752,251, filing date: August 17, 1971 Issue date: August 1973 Inventor: Edward G. Gaeke Assignee: General Motors Corporation.

Gonder, J. D. (2008). Route-based control of hybrid electric vehicles. In SAE World congress \& exhibition, April, Detroit, MI, USA, 2008-01-1315.

Gustafsson, F. (2000). Adaptive filtering and change detection. Chichester: Wiley.

Han, S., \& Rizos, C. (1999). Road slope information from GPS-derived trajectory data. Journal of Surveying Engineering, 125(2), 59-68.

Hellström, E. (2007). Look-ahead control of heavy trucks utilizing road topography. Licentiate thesis, Linköpings universitet, liU-TEK-LIC-2007:28, Thesis no. 1319.

Hellström, E., Ivarsson, M., Åslund, J., \& Nielsen, L. (2009). Look-ahead control for heavy trucks to minimize trip time and fuel consumption. Control Engineering Practice, 17(2), 245-254.

Johannesson, L. (2006). On energy management strategies for hybrid electric vehicles. Licentiate thesis, Chalmers tekniska högskola, series: R-Department of Signals and Systems, Chalmers University of Technology, no: R022.

Johannesson, L., \& Egardt, B. (2007). A novel algorithm for predictive control of parallel hybrid powertrains based on dynamic programming. In Fifth IFAC symposium on advances in automotive control, Monterey Coast CA, USA.

Kiencke, U., \& Nielsen, L. (2003). Automotive control systems. Berlin: Springer.

Lingman, P., \& Schmidtbauer, B. (2001). Road slope and vehicle mass estimation using Kalman filtering. In Proceedings of the 17th IAVSD symposium, Copenhagen, Denmark.

Mehra, R. K. (1970). On the identification of variances and adaptive kalman filtering. IEEE Transactions on Automatic Control, 15(2), 175-184.

Misra, P., \& Enge, P. (2006). Global positioning system: Signals measurements and performance (2nd ed.). Lincoln, MA: Ganga-Jamuna Press.

Oxford Technical Solutions Limited (2008). User manual; covers RT2000, RT3000 and RT4000 products. Oxford Technical Solutions Limited, 77 Heyford Park, Upper Heyford, Oxfordshire, England, 4 November 〈http://www.oxts.co.uk/down loads/rtman.pdf $>$.

Pettersson, N., \& Johansson, K. H. (2006). Modelling and control of auxiliary loads in heavy vehicles. International Journal of Control, 79(5), 479-495.

Rauch, H. E., Tung, F., \& Striebel, C. (1965). Maximum likelihood estimates of linear dynamic systems. AIAA Journal, 3(8), 1445-1450.

Sahlholm, P. (2008). Iterative road grade estimation for heavy duty vehicle control. Licentiate thesis, Kungliga Tekniska Högskolan, TRITA-EE 2008:056.

Sahlholm, P., Jansson, H., \& Johansson, K. H. (2007). Road grade estimation results using sensor and data fusion. In 14th world congress on intelligent transport systems, Beijing, China.

Sahlholm, P., Jansson, H., \& Johansson, K. H. (2008). Road grade estimation for lookahead vehicle control. In 17th IFAC world congress, Seoul, Korea.

Sahlholm, P., Jansson, H., Kozica, E., \& Johansson, K. H. (2007). A sensor and data fusion algorithm for road grade estimation. In Fifth IFAC symposium on advances in automotive control, Monterey Coast CA, USA.

Salmasi, F. (2007). Control strategies for hybrid electric vehicles: Evolution classification comparison and future trends. IEEE Transactions on Vehicular Technology, 56(5), 2393-2404.

Schroedl, S., Wagstaff, K., Rogers, S., Langley, P., \& Wilson, C. (2004). Mining GPS traces for map refinement. Data Mining and Knowledge Discovery, 9, 59-87.

Terwen, S., Back, M., \& Krebs, V. (2004). Predictive powertrain control for heavy duty trucks. In Proceedings of IFAC symposium on advances in automotive control, Salerno, Italy.

Vahidi, A., Stefanopolou, A., \& Peng, H. (2005). Recursive least squares with forgetting for online estimation of vehicle mass and road grade: Theory and experiments. Journal of Vehicle System Dynamics, 43, 31-57. 